\title{
Conventional and Dense Gas Techniques for the Production of Liposomes: A Review
}

\author{
Louise A. Meure, ${ }^{1}$ Neil R. Foster, ${ }^{1}$ and Fariba Dehghani ${ }^{2,3}$
}

Received 27 October 2007; accepted 24 March 2008; published online 3 July 2008

\begin{abstract}
The aim of this review paper is to compare the potential of various techniques developed for production of homogenous, stable liposomes. Traditional techniques, such as Bangham, detergent depletion, ether/ethanol injection, reverse-phase evaporation and emulsion methods, were compared with the recent advanced techniques developed for liposome formation. The major hurdles for scaling up the traditional methods are the consumption of large quantities of volatile organic solvent, the stability and homogeneity of the liposomal product, as well as the lengthy multiple steps involved. The new methods have been designed to alleviate the current issues for liposome formulation. Dense gas liposome techniques are still in their infancy, however they have remarkable advantages in reducing the use of organic solvents, providing fast, single-stage production and producing stable, uniform liposomes. Techniques such as the membrane contactor and heating methods are also promising as they eliminate the use of organic solvent, however high temperature is still required for processing.
\end{abstract}

KEY WORDS: dense gas technology; drug delivery; liposomes; nano-particles; phospholipid.

\section{INTRODUCTION}

Liposomes are formed when phospholipids spontaneously self-assemble into vesicles in the presence of water, producing an aqueous medium surrounded by a lipid membrane. Liposomes are excellent carrier systems for a variety of applications and are particularly ideal for drug delivery to the body due to the similarity to natural cells. The therapeutic index of drugs can be increased through incorporation into liposomes, which can act as a non-toxic, biodegradable system for solubilising drugs which have low aqueous solubility; protect rapidly degrading drugs from breakdown and consequently increase the drug residence time in the body; and also decrease drug toxicity (1). Delivery of drugs using liposomes also reduces the accumulation of drugs in sensitive tissues; alters the biodistribution and controls the release of an incorporated drug, therefore improving bioavailability; as well as targets the drug to specific tissue (1).

The stability and circulatory time in the bloodstream of liposomal formulations can be improved by addition of cholesterol and biocompatible, hydrophilic polymers with a flexible main chain, such as poly(ethylene glycol) $(2,3)$. Cholesterol is incorporated into the lipid bilayer and decreases any bilayer packing defects, therefore the structure is less prone to absorption and penetration by opsonins than

\footnotetext{
${ }^{1}$ School of Chemical Sciences and Engineering, The University of New South Wales, Sydney, New South Wales 2052, Australia.

${ }^{2}$ School of Chemical and Biomolecular Engineering, The University of Sydney, Sydney, New South Wales 2006, Australia.

${ }^{3}$ To whom correspondence should be addressed. (e-mail: fdehghani @usyd.edu.au)
}

vesicles with loosely packed bilayers (4). The structure of a liposome that has been stabilised through incorporating polymer into the particle surface is depicted in Fig. 1, with the polymer able to sterically hinder blood components from interacting with the liposome surface. A sufficiently long circulatory time in the bloodstream is required for a drug or carrier to find its target, thus the major medical application of sterically stabilised liposomes is in cancer therapy (5).

A manufacturing method is desirable to formulate liposomes with high entrapment efficiency, narrow size distribution, long-term stability and protective properties. The hurdles for broad use of liposomes for human applications are low stability, high cost for large scale production, toxicity of organic residue, and low entrapment efficiency. Pharmaceutical companies are eager to develop a technique to address the above issues. In this article, an overview of techniques used for manufacturing liposomes is provided. The advantages and drawbacks of each method, as well as its potential for bulk production of liposomes, are described and references have been provided for readers interested in acquiring further information for each process.

\section{BACKGROUND OF METHODS}

\section{Conventional Techniques for the Synthesis of Liposomes}

There are a wide variety of conventional techniques that can be used to produce liposomal formulations, including Bangham, detergent depletion, ether/ethanol injection, reversephase evaporation and emulsion methods $(6,7)$. All methods for producing liposomes require lipids to be combined by some means with an aqueous phase. The conventional methods generally involve a lipid solution being produced using an 


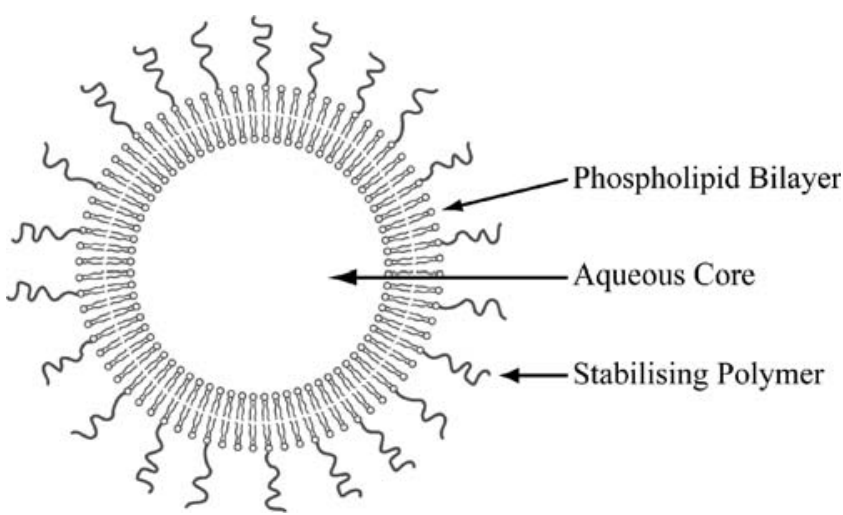

Fig. 1. A representation of the structure of a liposome, illustrating a lipid bilayer surrounding an aqueous core with stabilising polymer attached to the lipid surface

organic solvent prior to dispersion in the aqueous phase. The use of a volatile organic solvent may affect the chemical structure of an entrapped chemical and also contribute to the toxicity and stability of the liposome (8).

\section{Bangham Method}

The Bangham method is also known as the hand shaken or thin film hydration method and is one of the most widely used techniques for the formation of liposomes $(6,9,10)$. The process involves the dissolution of lipids in an organic phase, followed by the removal of the organic solvent, usually via evaporation, to form a lipid film. The solvent removal stage is time-consuming since it often requires several hours exposure to high vacuum. The final step is the dispersion or hydration of the lipid film with an aqueous media, carried out in conjuction with agitation to detach the swelling lamellae from the vessel surface and form sealed spherical structures. Liposomes produced via the Bangham method are often several microns in size. The Bangham method is one of the simplest methods for liposome formation, however it has limited use due to low entrapment efficiency, difficulty in removing organic solvent, homogenisation and small scale production.

Ran and Yalkowsky produced liposomes using the thin film method by replacing the organic solvent with halothane, a non-flammable, non-carcinogenic inhalation anaesthetic (11). Liposomes encapsulating an anti-tumour drug, both with and without polymer incorporation, were formed. After homogenisation by sonication at $45^{\circ} \mathrm{C}$, the liposomes ranged in size from 170-230 nm. High encapsulation efficiency and liposome stability were achieved using this method; with more than $90 \%$ of the drug remaining inside the liposomes after 3 months of storage at $4^{\circ} \mathrm{C}$.

\section{Detergent Depletion Method}

The detergent depletion method is a mild process for production of a wide variety of vesicle types and highly homogeneous liposomes. The method is based on the formation of detergent-lipid micelles, followed by the removal of the detergent to form liposomes. Detergent-lipid micelles can be formed by either hydrating a lipid with a detergent solution or by drying both lipid and detergent from an organic solution then adding an aqueous solution. The detergent associates with the lipid, protecting the hydrophobic sections from interacting with the aqueous phase, and thus micelles are formed rather than vesicles. The detergent is removed from the micellar solution, using dilution by 10 to 100 fold, dialysis, column chromatography or adsorption, to form the vesicles $(6,7,12)$. Despite dilution being the simplest method for detergent removal, the disadvantage is that the final concentration of liposomes in the solution is low and entrapment of any hydrophobic compounds is poor. The detergent also remains in the formulation and should be removed via other methods. The size and homogeneity of liposomes produced using detergent depletion are based on the rate at which the detergent is removed and the initial ratio of detergent to phospholipid (6). The method is very time consuming and equilibration of the micelles can be a slow process. Another potential disadvantage of the process is that the methods used to remove the detergent may also remove any other small hydrophilic compounds.

\section{Injection Methods}

The ethanol and ether injection methods involve the dissolution of the lipid into an organic phase, followed by the injection of the lipid solution into aqueous media, forming liposomes. The ethanol injection method was first described in 1973 by Batzri and Korn (13). The fine needle used to inject the lipid solution disperses the ethanol and, consequently, the phospholipid evenly in the aqueous media. The ethanol injection method is a simple method, but some lipids are poorly soluble in ethanol and heterogeneous liposomes are formed if adequate mixing is not achieved. Since the ethanol is injected directly into the aqueous media, residual solvent levels can be a concern unless post-formation removal is carried out.

The ether injection method differs from the ethanol injection method since the ether is immiscible with the aqueous phase, which is also heated so that the solvent is removed from the liposomal product $(14,15)$. The lipid-ether solution is injected slowly into the aqueous media and large unilamellar vesicles (LUVs) are formed. An advantage of the ether injection method compared to the ethanol injection method is the removal of the solvent from the product, enabling the process to be run for extended periods forming a concentrated liposomal product with high entrapment efficiencies. However, the slow injection means that it is a lengthy process. It has been suggested that injecting the ether solution at a rate faster than $0.2 \mathrm{ml} / \mathrm{min}$. can cause cooling of the aqueous media due to evaporation and that pre-evaporation of ether can cause nozzle blockages and the formation of multilamellar vesicles (MLVs) (6).

The ethanol injection method was used to prepare a camptothecin analogue (Gimatecan, an anti-cancer drug) liposome formulation (16). The concentration of the drug in the aqueous phase was increased by multiple injections of solution. The method is rapid, simple and reproducible for production of a ready-to-use liposome suspension. The particle size of liposomes produced by this method was a function of lipid nature and concentration, the drug to lipid ratio and the organic solvent and aqueous phase composition. 
Stano et al. (16) found that the multiple injection method allowed in situ preparation of liposome formulations with a large concentration of drug without altering the narrow size distribution. The major issue in the multiple injection method is the amount of ethanol in the final formulation and postformation processing (such as ultrafiltration) may be required to remove ethanol residue from the liposomal product.

Recent Modification Using an Inkjet. The inkjet method is a modern variation on the ethanol injection method and was developed by Hauschild et al. (17) for liposome formation with excellent control on particle size and high potential for scaling up. An inkjet printer was used to inject a phospholipid solution into water, producing small unilamellar vesicles (SUVs) with diameters in the range of 50-200 nm, narrow size distribution and efficient encapsulation of drugs into vesicles. Droplets with volumes in the range of picolitres were formed with high reproducibility using commercially available printers and cartridges.

Alcohol injection has been developed by two pharmaceutical companies: Alza and Polymun (18). Uniform liposomes are then produced using extrusion by the Alza Company and using a cross-injection module by Polymun. The alcohol injection method is a simple and a low energy process, however there are still issues associated with liposome stability due to residual solvent (18).

\section{Reverse Phase Evaporation Method}

The reverse-phase evaporation (REV) process was first described by Szoka and Papahadjopoulos (19) and is based on the formation of drops of water that are surrounded by lipid and dispersed in an organic solvent, referred to as inverted micelles. The technique is carried out by dissolving the lipids in an organic solvent, adding a small volume of aqueous phase, then sonicating the solution to produce inverted micelles. The organic solvent is removed using a rotary evaporator and a viscous gel forms. When sufficient solvent has been removed, the gel collapses and an aqueous suspension of vesicles forms. A disadvantage of the reverse-phase evaporation process, compared to some of the other conventional techniques, is that the compound to be encapsulated within the vesicles is in contact with an organic solvent, therefore the process is not suitable for fragile molecules such as peptides (7). It is possible to capture $30-45 \%$ of the aqueous phase within REVs and at optimal conditions, up to $65 \%$ entrapment may be achieved (19). The fact that a large portion of the aqueous volume is captured and a large aqueous space exists within the vesicle suggests that large macromolecules may be able to be encapsulated within REVs.

\section{Emulsion Method}

Emulsion methods for the production of liposomes generally involve the formation of a water-in-oil emulsion through the addition of a small amount of aqueous media to a larger volume of immiscible organic solution containing the phospholipid (6). The mixture is agitated to disperse the aqueous media as tiny droplets throughout the organic solvent and the lipid aligns itself into a monolayer at the boundary between the organic and aqueous phases. The size of the droplets is controlled by the agitation applied and the amount of lipid present, since there must be sufficient lipid to surround the droplet or it may fuse with other droplets (6).

The water-in-oil emulsion is transformed into a liposomal suspension through the formation of a double emulsion (20). The organic solution containing the water droplets is added to a large volume of aqueous media and agitated, producing a water-in-oil-in-water emulsion. A lipid monolayer also forms around the organic droplets producing aqueous cores surrounded by two lipid monolayers that are separated by an organic layer. Unilamellar liposomes with high entrapment of the initial aqueous media can then be formed by the removal of the organic solvent, for example, by passing a stream of nitrogen through the double emulsion (20).

A technique has been presented by Kim and Martin (21) for the production of multivesicular liposomes using a variation to the double emulsion method. The lipid, solvent composition and shaking time period are controlled so that multiple water droplets become contained within a single organic droplet in the double emulsion (6). Once the organic solvent is removed, the droplets form multiple compartments within a single lipid vesicle, usually 1-2 $\mu \mathrm{m}$ in diameter. Multivesicular liposomes provide the potential to deliver multiple compounds that are unstable when combined (6).

\section{Post-Formation Processing}

Multilamellar vesicles can be produced and then modified after production to alter the size, lamellarity or homogeneity and produce liposome populations with a specific size or property. The most common methods for post-formation processing are sonication, extrusion and high pressure homogenization $(6,7)$. A mechanical force is used to disrupt the large multilamellar vesicles into small membrane patches that ultimately form small unilamellar vesicles. For example, Takahashi et al. (22) developed an efficient process for the preparation of liposomes using a mechanochemical method. In this technique liposomes were formed by dispersing lecithins into deionised water using a homogeniser followed by a microfluidiser at 1,000 bar for $5 \mathrm{~min}$. Liposomes with particle size of $100 \mathrm{~nm}$ were formed from the combination of the homogeniser and microfluidiser with $80 \%$ drug loading efficiency (22). The aforementioned methods are generally size reduction techniques; however, as the liposomes are processed the lamellarity and heterogeneity are also decreased. Incorporation compounds can also be encapsulated within pre-formed liposomes through the use of remote loading, where a $\mathrm{pH}$, ion or proton gradient is induced across the lipid membrane $(23,24)$.

The conventional methods for liposome formation can produce vesicles with diameters ranging from $20 \mathrm{~nm}$ to several microns. Fan et al. (25) compared various liposome preparation methods such as thin film evaporation (Bangham method), sonication, reverse phase evaporation, melting and freezing-thawing. The highest encapsulating efficiency of liposomes for salidroside was achieved by freezing-thawing, followed by thin film evaporation, reverse phase evaporation, melting and then sonication. However, liposomal systems prepared by sonication, melting, and reverse phase evapora- 


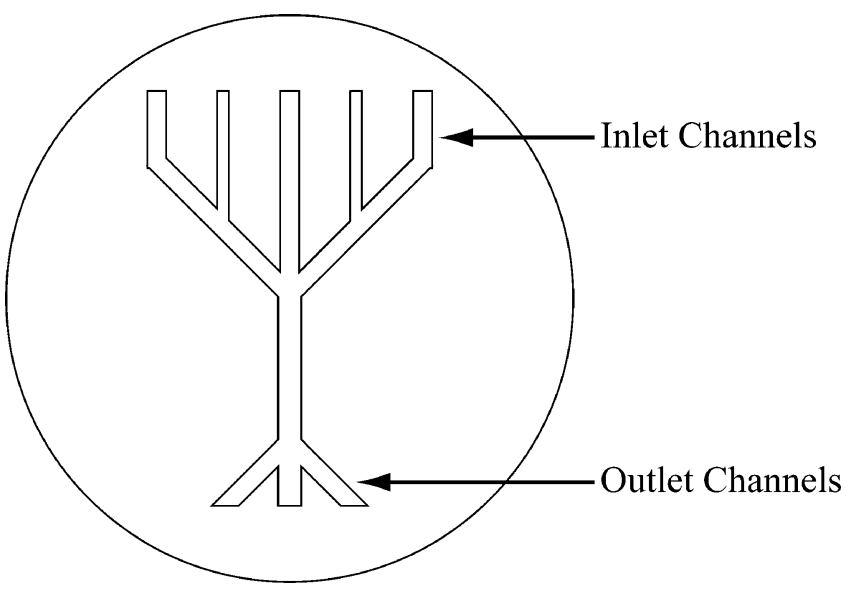

Fig. 2. Schematic diagram of the microfluidic channel used for liposome formulation by Jahn et al. (26)

tion displayed better dispersivity. Furthermore, salidroside liposomes prepared by melting had a better physicochemical stability (26).

Conventional methods are not suitable for processing fragile molecules because of the exposure to volatile organic solvents, detergent, sonication, or high shear homogenisation. The significant drawbacks of conventional preparation methods include being generally complex, time consuming, not easily scalable for mass production and there are difficulties in achieving high encapsulation efficiencies. Toxic solvent residue in the product is a significant disadvantage due to the cost of handling and removing solvents. Recently, unique methods were developed for producing liposomes to minimize the issues inherent in conventional liposome production techniques.

\section{RECENT METHODS DEVELOPED FOR THE SYNTHESIS OF LIPOSOMES}

\section{Freeze Drying of Monophase Solutions}

Li and Deng (18) recently designed a freeze drying of monophase solution method for formation of dry liposomes that can be stored for a long time in a sealed container. The process involved dissolving a phospholipid in $t$-butyl alcohol and water-sucrose to form an isotropic monophase solution and then freeze drying the solution. The freeze drying stage was performed by first freezing the sample at $-40^{\circ} \mathrm{C}$ for $8 \mathrm{~h}$, followed by drying at this temperature for 48 hours and finally drying the product at $25^{\circ} \mathrm{C}$ for $10 \mathrm{~h}$, which is very time consuming.

$\mathrm{Li}$ and Deng observed that the size and polydispersity of liposome particles were decreased by increasing the sucrose concentration. This effect may result from enhancing the viscosity of sucrose solutions by increasing the concentration, which inhibits the appearance of large solid t-butyl hydrate, consequently forcing the formation of lipid-rich minidomains (18). Furthermore, sucrose might prevent the fusion of different minidomains during the drying process and in dry status. The particle size of liposomes fabricated by this freeze drying method was within 100 to $200 \mathrm{~nm}$, which was dramatically smaller and more uniform than the micron range of liposomes produced previously using a t-butyl alcohol- water mixture in the absence of sucrose (18). The $\mathrm{Li}$ and Deng method can be used for both lipophilic and hydrophilic drugs; however, the t-butyl alcohol-water system without sucrose was used only for lipophilic drugs that are soluble in $t$-butyl alcohol.

\section{Microfluidic Channel Method}

Jahn et al. (26) developed a microfluidic method for controlled liposome formation. Rectangular microfluidic channels with a depth of $100 \mu \mathrm{m}$ and a width of 46 or $64 \mu \mathrm{m}$ were fabricated in a silicon wafer, as shown in Fig. 2. Nanoports were bonded to the backside of the silicon wafer and were connected to a syringe via a $254 \mu \mathrm{m}$ capillary tube. A lipid solution was injected into the central channel of the microfluidic network while aqueous solutions passed into the side channels, intersecting with the central channel. Liposomes can be formed as different shear forces are generated at the liquid interfaces by the changing flow rate ratio. The process involves a stream of lipid dissolved in alcohol passing between two aqueous streams in a microfluidic channel, with mixing occurring at the liquid interfaces and thus liposomes forming. The laminar flow in the channels enables control over the diffusive mixing and thus the size and size distribution of the liposomes can be manipulated through adjusting the flow conditions. Jahn et al. (26) demonstrated that monodispersed liposomes can be produced by adjusting the flow rates of the phospholipid and water solutions in microchannels. Decreasing the sample stream width to micrometer length scales allows for intimate mixing and uniform conditions for formation of liposomes with a mean geometric radius of $29 \mathrm{~nm}$. Liposome self assembly by this microfluidic method can be used for drug encapsulation immediately prior to use, hence eliminating shelf life limitations of the current liposome preparation techniques (26).

\section{Membrane Contactor Method}

Charcosset et al. (27) designed a new process for solid lipid nanoparticle formation that consisted of a membrane contactor, which can be used for large scale production of liposomes. In this method, a lipid phase was pressed at temperatures above the melting point of the lipid through a membrane with a specified pore size. Nitrogen gas at pressures below 6 bar was sufficient for passing the molten phase through the membrane. Solid lipid nanoparticles with particle size between 70 and $215 \mathrm{~nm}$ were formed and collected by recirculation of a cold stream of aqueous phase inside the membrane module. The advantages of this new process are its simple design, the control of the solid-lipid nanoparticle size by tuning the process parameters and its scaling-up abilities (27).

\section{Heating Method}

A heating method developed by Mozafari to produce liposomes involves the hydration of a phospholipid in an aqueous solution containing 3 vol\% glycerol and increasing the temperature to $60^{\circ} \mathrm{C}$ or $120^{\circ} \mathrm{C}(8)$. Glycerol is utilized since it is a water soluble and physiologically acceptable chemical with the ability to act as an isotonising agent and 


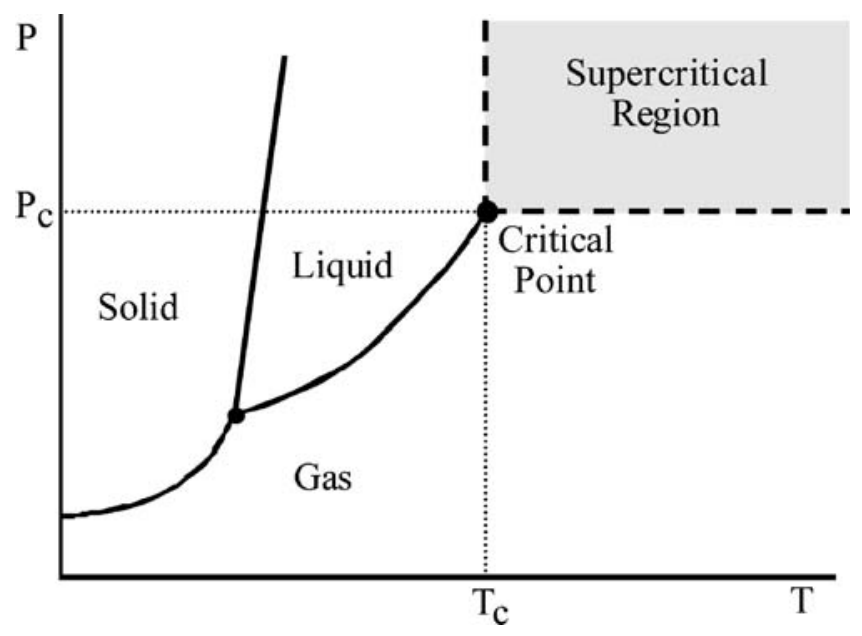

Fig. 3. Pressure-temperature diagram for a pure component

increase the stability of lipid vesicles due to preventing coagulation and sedimentation. No degradation of lipid was reported for liposomes fabricated by the heating method and no need for sterilisation as high temperature was used in this technique. A drug was incorporated into liposomes using the heating method by adding the drug to the solution at different stages: 1) at the beginning; 2) when the temperature was above the transition temperature of the lipids; and 3) at ambient temperature after liposome preparation, for heat sensitive materials such as DNA (8).

\section{Dense Gas Techniques}

Dense gas processes have been developed for the production of liposomes in order to eliminate the issues involved in conventional preparation. The term dense gas is a general expression used to refer to a substance in the region surrounding the critical point. The phase diagram for a pure component is depicted in Fig. 3 and shows the liquid, gas and supercritical fluid regions meeting at the critical point. Dense gases possess solvent power similar to that of liquids along with mass transport properties similar to those of gases. The unique properties of dense gases have been exploited to replace many organic solvents and enable improved processing techniques, in particular separation, purification and size reduction processes. The most widely used dense gas is carbon dioxide since it is non-flammable, non-toxic, non-corrosive, inexpensive, environmentally acceptable and has easily accessible critical parameters of $31.1^{\circ} \mathrm{C}$ and 73.8 bar. The low critical temperature of $\mathrm{CO}_{2}$ means that a low energy input is required for dense gas processing and the solvent is suitable for use with thermally labile materials. The solvent can be easily recovered after processing by simply returning to atmospheric pressure.

Dense gas processing can provide sterile operating conditions and one-step production that can alleviate the current liposome sterilisation issues $(28,29)$. Currently, sterilised liposomes are formed by the use of sterilised solutions and equipment during liposome manufacturing, which is time consuming (30,31). Many other sterilisation methods are unsuitable since liposomes are thermally labile and hydrolysed by $\gamma$-radiation. Sterilisation methods that involve chemicals or heating can also release the encapsulated material. Filtration is a non-destructive liposome sterilisation method, however it is very time consuming and not efficient for removing viruses or bulk production.

\section{Supercritical Fluid Injection and Decompression Method}

The first dense gas techniques for the formation of liposomes, referred to as the injection and the decompression methods, were described by Castor and Chu in 1994 (32). In the injection method, a mixture of lipid, organic co-solvent and compressed gas is injected through a nozzle into an aqueous solution. Alternatively, the decompression method involves a mixture of lipid, organic co-solvent, compressed gas and aqueous solution being decompressed through a nozzle to form liposomes. A simplified representation of the apparatus used in the injection and decompression methods is given in Fig. 4. The major distinction between the processes is the incorporation of the aqueous solution. In the injection method the compressed phase is sprayed into water, whereas in the decompression method, the aqueous phase is incorporated into the compressed phase, which is sprayed into air. The rate of depressurisation influences the size of the liposomes formed. Castor and Chu claim the injection and decompression processes are capable of producing sterile, pharmaceutical grade liposomes of a pre-determined size that have a narrow particle size distribution and are substantially solvent free (30). It is preferred that any pharmaceutical incorporated is not shear sensitive when applied to the decompression method (31). Conversely, the injection method does not subject the materials to high shear forces and so is suitable for fragile drug molecules. A homogenisation or size-reduction method was also described by Castor and Chu through depressurising a mixture of pre-formed MLVs and compressed fluid so as to separate the fluid and produce liposomes of a uniform size $(30,32,33)$.

In 2005, Castor (34) reported the production of small uniform liposomes (or phospholipid nanosomes) using processes similar to the injection and decompression methods and referred to them as the SuperFluids phospholipid nanosome (SFS-CFN) manufacturing process. Castor states that a key ingredient of the process is the ability of the dense gas to dissolve the materials and consequently utilizes co-solvents and a circulation loop to ensure the solutes are completely dissolved prior to depressurisation. A number of variations on the operation of the process were investigated based on the type of incorporation compound and its subsequent solubility in the aqueous or dense gas phase. A hydrophilic protein (cytochrome-C) and hydrophobic model drugs (paclitaxel, camptothecin and betulinic acid) were encapsulated within the liposomes and in vitro and in vivo testing was conducted on the product (34).

The data presented by Castor suggests that the SFS-CFN experiments produced liposomes in the size range $100 \mathrm{~nm}$ and $4 \mu \mathrm{m}$, but more commonly less than $200 \mathrm{~nm}$ at $60^{\circ} \mathrm{C}$ and 280 bar (34). Liposome formulations produced by this method were stable, in terms of particle size distribution, for 6 months when stored at $4^{\circ} \mathrm{C}$. The in vivo study of the liposomes containing paclitaxel showed that the formulation had a significantly greater effect on the cancer cells than the conventional therapeutic formulation and may therefore provide improved treatment of breast cancer (34). 


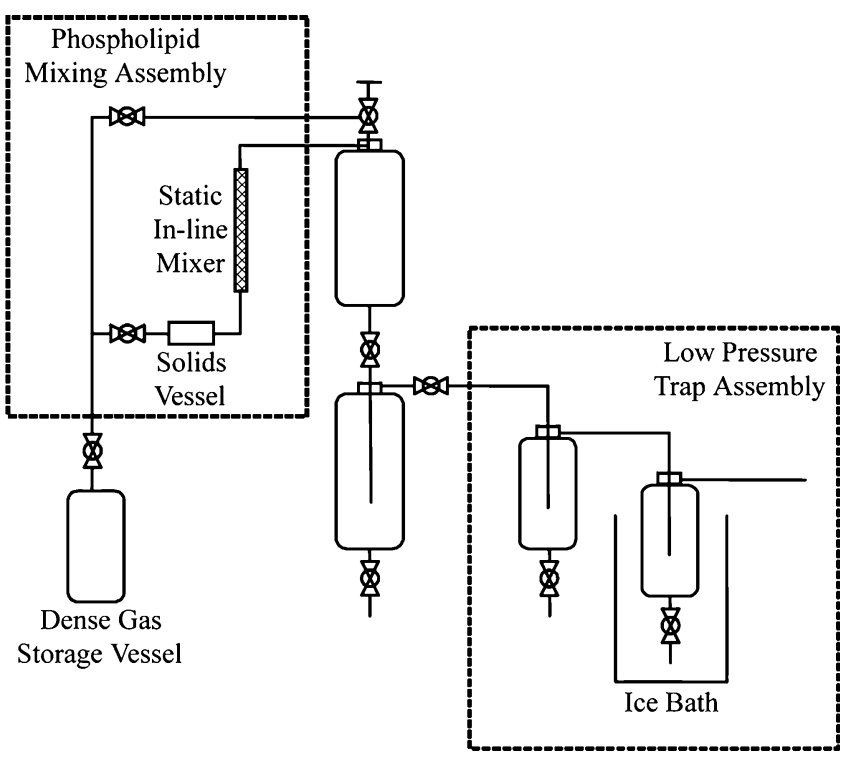

Fig. 4. Simplified schematic of the apparatus used in the Decompression and Injection method (30)

The SFS-CFN process utilizes dense gases, polar cosolvents and elevated temperatures and pressures to achieve solubilisation of the raw materials for liposome processing. The use of solvents such as chloroform is eliminated in the SFS-CFN process, however the processing time, temperature and pressure (350 bar) required for the process are still high (34).

\section{Supercritical Fluid Liposome Method}

Frederiksen et al. described the supercritical liposome method in 1994, which is similar to the injection method developed by Castor and $\mathrm{Chu}$, and produced SUVs with particle size between 20 and $50 \mathrm{~nm}(28,35,36)$. The process is depicted in Fig. 5 and involves the dissolution of phospholipid and cholesterol into supercritical carbon dioxide at $60^{\circ} \mathrm{C}$ and 250 bar using $5-7 \%$ ethanol as a co-solvent. The lipid and cholesterol were dissolved after being placed in a cartridge through which repeated cycles of carbon dioxide/ethanol was passed. The recycling system was used to dissolve both the lipid and cholesterol, which have different solubilities in the dense gas phase, such that homogeneous liposomes could be generated. The solution was then rapidly expanded into an aqueous phase containing the hydrophilic compound to be entrapped.

The foam formation that may appear in the process upon depressurisation of the high pressure solution directly into the aqueous phase was eliminated by expanding and simultaneously contacting the pressurised solution with the aqueous solution in a capillary prior to dilution in a low-pressure recycling system. An encapsulation efficiency of $15 \%$ was achieved using this method (35). The formation of the liposomes in the capillary is a significant difference between the supercritical liposome method and the injection method. In addition to SUVs, a small fraction of large MLVs $(250 \mathrm{~nm})$ were also formed (35). The dimensions of the capillary within which the liposomes are formed affects the encapsulation efficiency and the size of the liposomes, therefore the internal surface area of the capillary should be minimized to achieve a high encapsulation volume. The amount of ethanol required to produce liposomes by the ethanol injection method was 15 fold more than that required for the supercritical liposome method (35). However, the encapsulation efficiency was $20 \%$ for the supercritical liposome technique, which was $50 \%$ lower than that achieved using conventional liposome formation techniques. It is believed that the lower encapsulation efficiency is compensated for by the elimination of the requirement for toxic solvents, the decreased organic solvent consumption and the convenient scaleable method.

Bridson et al. also studied the production of liposomes using a method similar to Frederiksen et al. (35-37). The focus of the study was on investigating the entrapment capabilities and the effects of process parameters on the product obtained. Bridson et al. produced liposomes with between three and five bilayers and an average diameter of $200 \mathrm{~nm}$, which is significantly different to the $20-50 \mathrm{~nm}$ SUVs reported by Frederiksen et al. The authors concluded that liposomes with a broad particle size distribution were produced and post-formation processing may be required to improve the product.

\section{Supercritical Reverse Phase Evaporation Process}

In the Supercritical Reverse Phase Evaporation (scRPE) method developed by Otake et al., the lipid, organic cosolvent and compressed gas are combined in a stirred, variable volume cell at a temperature above the lipid phase transition temperature, as depicted in Fig. 6 (29). An aqueous solution is then slowly introduced to the cell. The pressure is reduced by the release of the compressed gas and liposomes are formed. The principle of the scRPE method is similar to the decompression method described by Castor and Chu since the lipid, aqueous phase, dense gas and modifier are combined and then depressurised to form liposomes. The difference is that the depressurisation generally occurs in the decompression method by spraying the solution through a nozzle, whereas in the scRPE method the depressurisation occurs by the release of the dense gas from a variable volume cell containing the materials. The process described by Otake et al. is physically simpler than Frederiksen et al.'s supercritical liposome method and requires less carbon dioxide, but the mechanism of formation is more complicated $(29,35,36)$. The liposomes produced by scRPE were also significantly larger than those produced by Frederiksen et al. $(29,35,36)$.

In the scRPE method, a water $/ \mathrm{CO}_{2}$ microemulsion forms as water is introduced into the cell, then when more water is introduced the cell becomes opaque as a macroemulsion forms, based on reverse micelles of lipid (29). A transition occurs from a water $/ \mathrm{CO}_{2}$ emulsion to a $\mathrm{CO}_{2} /$ water emulsion with increasing amounts of aqueous phase. Liposomes may then be formed as the $\mathrm{CO}_{2}$ is evaporated from the aqueous phase upon depressurisation. Attempts were made to produce liposomes when some solid lipid was present, rather than completely dissolved lipid, however the trapping efficiencies achieved were extremely low (29). The lipids used have very low solubility in carbon dioxide; therefore the organic co-solvent is incorporated to improve the solubility of the lipid in the dense gas phase. Although organic co-solvent is used in the scRPE method, non-toxic solvents can be 


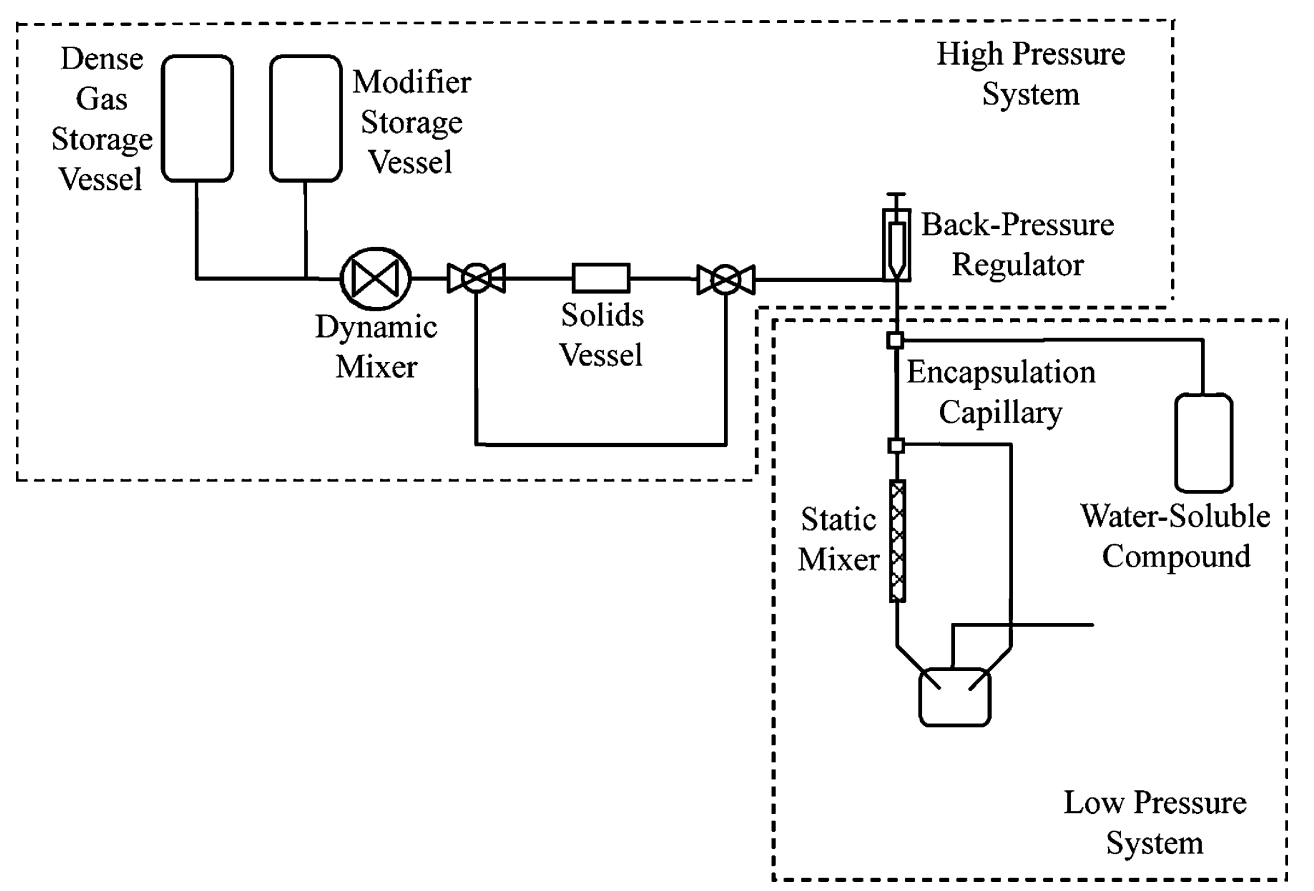

Fig. 5. Simplified schematic of the apparatus used in the supercritical liposome method (39)

utilized, such as ethanol. Similar to that found by Frederiksen et al., the optimum concentration of ethanol added was $7 \mathrm{wt} \%$ when high temperatures and pressures were used $\left(60^{\circ} \mathrm{C}\right.$ and 200 bar) (29).

Otake et al. formed ellipsoidal LUVs that ranged in size from $100 \mathrm{~nm}-1.2 \mu \mathrm{m}$ and the vesicles became spherical as the size was decreased $(29,38)$. It was suggested that the ellipsoidal shape is caused by the lower stability of the LUVs in comparison to spherical MLVs. A high trapping efficiency was achieved for encapsulation of both water-soluble (glucose) and oil-soluble (cholesterol) materials (29). The oilsoluble substance was present with the lipid and the watersoluble substance introduced with the aqueous phase. The

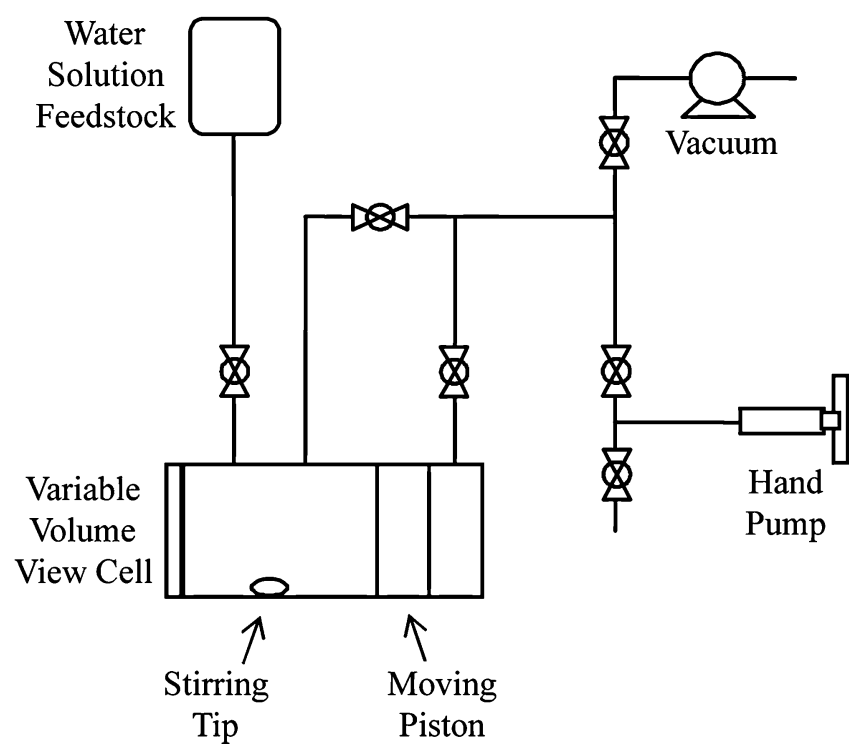

Fig. 6. Simplified representation of the apparatus used in the scRPE method (33) product from the scRPE method was compared to MLVs, LUVs and giant unilamellar vesicles (GUVs) formed using conventional methods $(29,39)$. The scRPE method achieved a trapping efficiency comparable to the conventional LUVs, lower than the GUVs and higher than the MLVs. scRPE has been carried out using a semi-batch apparatus that is capable of producing $50 \mathrm{~cm}^{3}$ of liposomal product in $100 \mathrm{~min}(40)$. Water-soluble vitamin $\mathrm{C}$ and oil-soluble cholesterol, ceramide 3 and a vitamin A derivative were encapsulated in liposomes using this technique.

Otake et al. (41) recently developed a new method known as the improved supercritical reverse phase evaporation (ISCRPE) technique to avoid the use of organic solvent in liposome formation and enhance the stability and the drug loading efficiency. In this method, the inhomogeneous mixture of a phospholipid powder and aqueous solution was prepared using a stirrer, the solution was then pressurised by $\mathrm{CO}_{2}$ to form a $\mathrm{CO}_{2} /$ water emulsion at $60^{\circ} \mathrm{C}$ and 200 bar, and after $40 \mathrm{~min}$ the system was depressurised to generate liposomes. Eliminating ethanol from the scRPE process decreases the liposome disruption and enhances its trapping efficiency. The rate of phase inversion during depressurisation has an impact on the properties of the liposomes. The trapping efficiency and the size of the liposome were increased at slow depressurisation rate, as a result of decreasing the surface-to-volume ratio of particles with the same amount of phospholipid. Otake et al. (41) also observed that the trapping efficiency was promoted by increasing the phospholipid chain length and addition of a double bond into the main chain.

The liposome formulation prepared by the ISCRPE technique remained stable after 30 days, while products from the Bangham and scRPE methods were not stable after $6 \mathrm{~h}$ and 14 days, respectively (41). It was also found that the stability of liposomes produced from ISCRPE with $\mathrm{CO}_{2}$ was higher than with $\mathrm{N}_{2}$, possibly due to the static repulsion of the 
carbonic acids incorporated into the bilayer membrane. The addition of chitosan to the liposomal formulation in the ISCRPE method did not affect its stability (30 days) and a trapping efficiency of $17 \%$ was achieved (42).

\section{Other Dense Gas Processes}

Work has been carried out on the formation of lipid particles, as a step towards liposome formation, using dense gas technology. Magnan et al. micronized soy lecithin using two dense gas processes, the supercritical anti-solvent (SAS) precipitation process and rapid expansion of supercritical solutions (RESS) process, and claimed that future work would deal with the formation of liposomes through the dispersion of the fine phospholipid particles in water, which could be conducted in the same vessel without opening $(43,44)$. In the SAS process, ethanol was used to dissolve the soy lecithin and carbon dioxide was used as the antisolvent for precipitation. Fine, but aggregated, phospholipid particles were formed that ranged in size from $1-40 \mu \mathrm{m}$ and contained no residual solvent (43). The higher the percentage of the phosphatidylcholine in soy lecithin, then better solubility and yield could be achieved.

The RESS process uses a dense gas, with or without cosolvents such as ethanol, to solubilise the lipid before depressurisation to form microparticles. The RESS process was not successful for liposome formulation, due to the inability to separate the lipid particles from the co-solvent upon depressurisation, utilising $\mathrm{CO}_{2}$ as an anti-solvent for micronization of liposomes from ethanol solution was efficient (44). Further information about phase behaviour, masstransfer kinetics as well as hydrodynamic considerations are required for liposome formulation by the SAS and RESS techniques. It is clearly evident, however, that there is a great amount of interest in lipid and liposome processing using dense gas techniques.

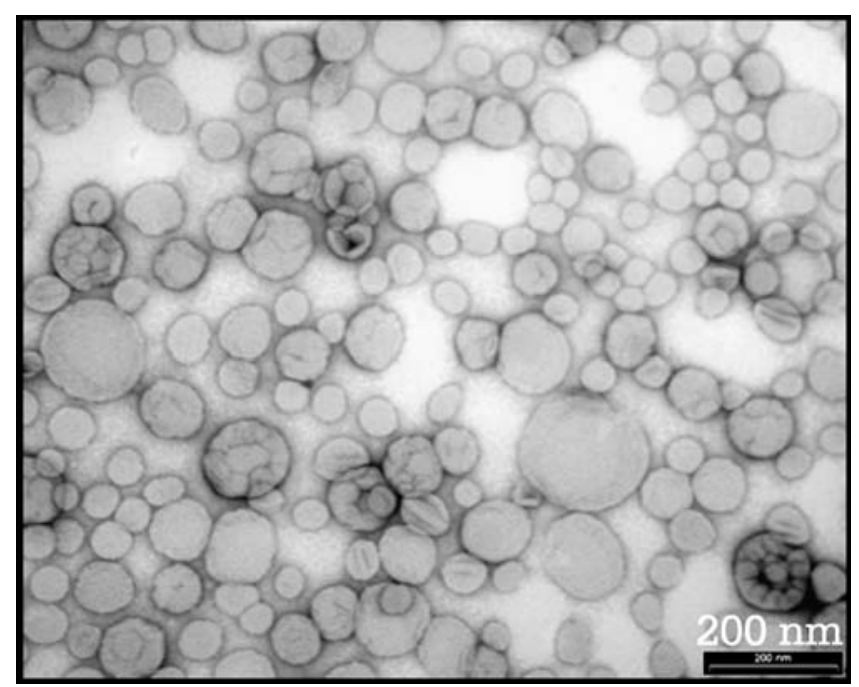

Fig. 8. Liposomes produced by a new rapid, simple, one-step dense gas liposome formation process, DESAM (55)

More recently, Kunastitchai et al. micronized phosphatidylcholine, cholesterol and miconazole using the aerosol solvent extraction system (ASES), a process essentially identical to the SAS process, and referred to the product as liposomes in a dry and reconstitutable form $(45,46)$. Miconazole was used as the model drug and poloxamer 407 was incorporated in some experiments as a surfactant. A methanol and methylene chloride mixture was used to solubilise the components prior to processing, however 3-4 h of washing was required postformation to minimize the amount of residual solvent left in the product. Aggregates of microparticles were produced that ranged in size from a few microns to $40 \mu \mathrm{m}$. The particles were added to phosphate buffer at $55^{\circ} \mathrm{C}$ and agitated by vortex mixing to hydrate the microparticles and produce liposomes.

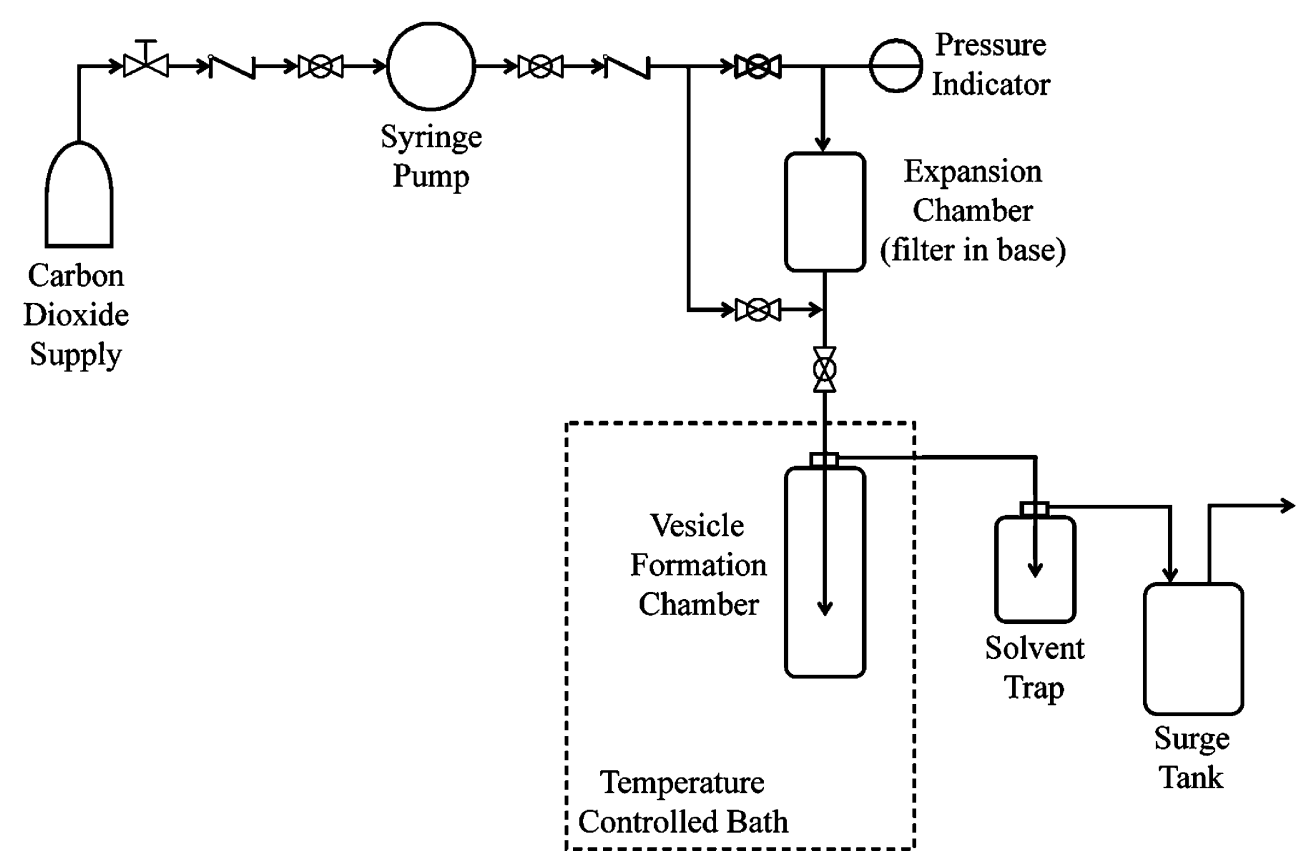

Fig. 7. Schematic diagram of the apparatus used in the DESAM process (55) 
Table I. Advantages and Disadvantages for Large Scale Production using the Major Conventional Liposome Formation Methods

\begin{tabular}{|c|c|c|}
\hline Conventional Methods & Advantages & Disadvantages \\
\hline Bangham & Simple process & $\begin{array}{l}\text { Large amount of organic solvent, } \\
\text { requires vigorous agitation, large vesicles with } \\
\text { no control on particle size, time consuming, } \\
\text { sterilisation issue }\end{array}$ \\
\hline Detergent depletion & $\begin{array}{l}\text { Simple process, homogenous product, } \\
\text { control of particle size }\end{array}$ & $\begin{array}{l}\text { Large amount of organic solvent, } \\
\text { detergent residue in the product, } \\
\text { time consuming, poor entrapment efficiency, } \\
\text { low yield, sterilisation issue }\end{array}$ \\
\hline Ethanol/ether injection & Simple process & $\begin{array}{l}\text { Organic solvent residue, nozzle } \\
\text { blockage in ether system due to } \\
\text { pre-evaporation, time consuming, } \\
\text { sterilisation issue }\end{array}$ \\
\hline Inkjet injection & $\begin{array}{l}\text { Simple process, control on particle size and homogeneity, } \\
\text { high potential for scale-up and encapsulation efficiency, } \\
\text { low energy process }\end{array}$ & $\begin{array}{l}\text { Organic solvent residue, low drug } \\
\text { stability, sterilisation issue }\end{array}$ \\
\hline Reverse phase evaporation & Simple design, reasonable encapsulation efficiency & $\begin{array}{l}\text { Not suitable for encapsulation of fragile } \\
\text { molecule due to large quantity of } \\
\text { organic solvent use, time consuming, } \\
\text { sterilisation issue }\end{array}$ \\
\hline Emulsion & $\begin{array}{l}\text { Simple, potential to fabricate multivesicular liposome } \\
\text { for delivery of multiple compounds that are not } \\
\text { stable in combination }\end{array}$ & $\begin{array}{l}\text { Large amount of organic solvent, requires } \\
\text { vigorous agitation, sterilisation issue }\end{array}$ \\
\hline
\end{tabular}

Bridson et al. produced finely divided phospholipid powders by using compressed carbon dioxide as an antisolvent for precipitating lipid from ethanol solution (37). In the SAS and ASES processes, the lipid-alcohol solution is sprayed via a nozzle into a chamber containing the dense gas anti-solvent. However, Bridson et al. injected the lipid-alcohol solution into a T-piece through which the dense gas was flowing then collected the micronized lipid downstream in a filter. The phospholipid powder was hydrated by agitation in an aqueous solution at a temperature above the glass transition temperature of the lipid, forming liposomes of approximately $5 \mu \mathrm{m}$ diameter. It is clearly evident that the size of the liposomes produced by firstly micronizing lipid was dramatically larger than liposomes produced by most other dense gas formation techniques.

The disadvantage of processes that produce micronized lipid as an initial step in liposome production is the requirement for multiple stages to achieve the final liposomal product. However, Kunastitchai et al. (45) suggest that producing lipid particles instead of liposomes is beneficial since the stability of liposomes during storage can be an issue and lipid particles can be rehydrated to liposomes prior to use. Therefore, the liposome production is still being conducted in two stages, however there is a time delay between the stages such that the liposomes are only formed immediately prior to use.

Table II. Advantages and Disadvantages for Large Scale Production using the Major Advanced Liposome Formation Methods

\begin{tabular}{|c|c|c|}
\hline Advanced Methods & Advantages & Disadvantages \\
\hline Freeze drying of monophase solution & $\begin{array}{l}\text { Feasible to process dry liposome, low organic } \\
\text { solvent residue, increased stability }\end{array}$ & Time consuming, sterilisation issue \\
\hline Microfluidic channel & $\begin{array}{l}\text { Control of particle size, allows production of vesicles } \\
\text { with diameter up to } 29 \mathrm{~nm}\end{array}$ & $\begin{array}{l}\text { Not suitable for bulk production, } \\
\text { organic solvent use, issues with } \\
\text { availability and high cost of } \\
\text { microfluidic channels }\end{array}$ \\
\hline Membrane contactor & $\begin{array}{l}\text { Simple process for controlled particle size, no use } \\
\text { of organic solvent }\end{array}$ & $\begin{array}{l}\text { High temperature, membrane } \\
\text { blockage and sterilisation issues }\end{array}$ \\
\hline Heating & $\begin{array}{l}\text { Simple, no organic solvent use, in situ sterilisation, } \\
\text { scale-up possible }\end{array}$ & $\begin{array}{l}\text { Use of high temperature that } \\
\text { may not be cost effective for } \\
\text { large scale }\end{array}$ \\
\hline $\begin{array}{l}\text { Supercritical fluid injection } \\
\text { and decompression }\end{array}$ & $\begin{array}{l}\text { Control of particle size, possible in situ sterilisation, } \\
\text { low organic solvent consumption }\end{array}$ & $\begin{array}{l}\text { High capital cost, low yield, high } \\
\text { pressure up to } 350 \text { bar used }\end{array}$ \\
\hline Supercritical liposome method & Possible in situ sterilisation, low organic solvent consumption & $\begin{array}{l}\text { High capital cost, low yield and } \\
\text { encapsulation efficiency, high } \\
\text { pressure up to } 250 \text { bar used }\end{array}$ \\
\hline $\begin{array}{l}\text { Improved/ supercritical reverse } \\
\text { phase evaporation }\end{array}$ & $\begin{array}{l}\text { No need for using nozzles, one-step closed vessel } \\
\text { production, low organic solvent consumption, rapid process, } \\
\text { scale-up potential, enhance stability by eliminating ethanol }\end{array}$ & $\begin{array}{l}\text { High capital cost, high pressure } \\
\text { up to } 200 \text { bar used }\end{array}$ \\
\hline $\begin{array}{l}\text { Depressurisation of an Expanded } \\
\text { Solution into Aqueous Media }\end{array}$ & $\begin{array}{l}\text { Simple and rapid process, scale-up potential, low temperature } \\
\text { process, in situ removal of organic solvent residue }\end{array}$ & High capital cost \\
\hline
\end{tabular}


Kunastitchai et al. (46) studied the stability of both the lipid-drug particles and reconstituted liposomes by examining the drug loading initially and months later. The stability showed varying trends depending on composition, storage temperature and $\mathrm{pH}$ of hydration medium. The data presented by Kunastitchai et al. suggests that the micronized particles and the liposomes hydrated at $\mathrm{pH} 7.2$ were generally stable for the length of the stability study (3-5 months), based on drug loading. The particle size distribution also showed good stability over 3 months for the reconstituted liposomes when a pH 7.2 hydration solution was used. Lastly, Kunastitchai examined in vitro cellular uptake of the liposomes by incubation with human lymphocytes and claimed that the liposomes appear to be substantially internalised, possibly leading to degradation of the structure and release of the drug (46).

During the last few years a few patents have been filed using dense gas technology for liposome formulation that include minimal or no organic solvents (47-54). Wada and Motokui combined lipid, cholesterol, a lipid-polymer compound and a water soluble agent with supercritical carbon dioxide $(52,53)$. The pressure was then reduced, releasing the carbon dioxide, and the dispersion mixed with a higher concentration solution of water soluble agent. The method was used to encapsulate x-ray contrast agents within liposomes. Ueda et al. used a dense gas emulsion process to encapsulate photosensitizers or imaging, anti-cancer, or antifungal agents in liposomes $(47,51)$. The processes described are similar to the scRPE method described earlier by Otake et al. (29). Ueda also encapsulated gas into liposomes through using pressure difference (50).

Despite the clear advantages of dense gas liposome production, there are also problems with the known processes for liposome formation. The dense gas processes described to produce liposomes directly generally require elevated pressures of at least 69 bar $(1,000 \mathrm{psi})$ and the conditions commonly used are 200-300 bar and temperatures of $60^{\circ} \mathrm{C}$. Solubilising the materials in a dense gas and establishing the appropriate conditions for solubilisation can also be a complicated process. It is difficult working with mixtures in supercritical and near critical systems in conditions where solid components of the mixture readily block nozzles and other parts of the equipment used. An additional drawback is that the existing dense gas processes for producing liposomes do not provide a way for the co-solvent or modifier that is mixed with the dense gas to be removed from the system, with the exception of the ISCRPE process. Therefore, the presence of the solvent or modifier in the liposomal product becomes a contaminant and consequently a potential problem.

Accordingly, investigations have been carried out by the authors to develop a process to form liposomes which utilizes the advantages of dense gases without the disadvantages described above. The rapid, one-step process (depicted in Fig. 7) involves the depressurisation of expanded solution into aqueous media (DESAM) and was used to produce uniform liposomes and remove organic solution in situ (55). In particular, the process was designed to utilize moderate temperatures and pressures below 60 bar. The new method needed to be both easily adaptable to a variety of incorporation compounds and easily scalable for mass production. Liposomes with diameters generally between $50-200 \mathrm{~nm}$ have been successfully produced, as shown in Fig. 8. The process was also extended to the formation of sterically stabilised liposomes.

\section{Large Scale Liposome Fabrication}

Various techniques have been developed for the bench scale liposome formation, however the broad application of liposomes in drug delivery is still impeded due to scale up issues. The advantages and disadvantages, in regard to the transition from lab to large scale operation, of the major liposome processes described above are summarised in Table I for conventional and in Table II for the recent advanced methods. Liposome formation processes should be validated according to Good Manufacturing Practice (GMP) protocols prior to commercialisation. The validation of current methods for liposome formation is challenging as there are still issues in reproducible production of desired size vesicles, the use of organic solvents in the process, stability of formulation, multiple-stage processing and problems associated with current liposome sterilisation. Liposomal drug formulations have been increasingly used in clinical trials during the last few years with many encouraging results. The outcome of the clinical trials transferred liposome from laboratory to clinical applications. It is therefore of particular importance to design a process that is cost effective for production of liposomes in large quantities for new drug formulations.

Wagner et al. (56) presented a new scalable liposome production scheme for the ethanol injection technique that allowed production on a several litres scale. In this process a cross-flow injection module was used that allowed liposomes to be manufactured regardless of production scale and no mechanical forces were required to generate homogenous liposomes. The size of liposome vesicles and encapsulation rate were controlled by the lipid concentration at the injection point, injection pressure and injection rate. The drug-liposome formulation was first optimized in a laboratory scale and the scale-up was performed by changing the size of the process vessel. Regardless of the advantages of this method compared with other liposome formation techniques, there is still the issue of residual ethanol in the final product (56).

New, advanced technology, such as using microfluidic devices and membranes, could be implemented for point-ofcare formulation preparation, thus eliminating stability limitations on the product. The scale up of the aforementioned processes will, however, rely on fabrication of low-cost microfluidic devices and advances in membrane technology. The dense gas techniques developed can address scale up issues for liposome formulation, in particular enabling production conducted in a totally enclosed single step. There is also evidence that carbon dioxide can inactivate bacteria and endotoxins, which is beneficial in terms of GMP regulations, but does not denature the bio-molecules (57-59).

\section{CONCLUSIONS}

Liposomes have wide potential in the pharmaceutical industry, however companies are interested in using a low cost, simple, one-step process that complies with GMP restrictions. Many processes are under development to address the major issues in liposome processing, enhance 
stability of the product and eliminate organic solvent usage. Despite the drawbacks of conventional methods, large scale liposome manufacture has been carried out using recent advances in injection methods. The ISCRPE and DESAM methods, as well as application of microfluid channels and membranes, are promising in terms of process intensification (decreasing the processing steps, time, complexity) and production of sterile, homogenous, stable liposomal formulation products.

\section{REFERENCES}

1. T. M. Allen, and E. H. Moase. Therapeutic opportunities for targeted liposomal drug delivery. Adv. Drug Delivery Rev. 21 (2):117-133 (1996).

2. S. S. Chrai, R. Murari, and I. Ahmad. Liposomes (a review). Part two: Drug delivery systems. BioPharm. 15(1):40,42-43,49 (2002).

3. V. P. Torchilin, and V. S. Trubetskoy. Which polymers can make nanoparticulate drug carriers long-circulating? Adv. Drug Delivery Rev. 16(2-3):141-155 (1995).

4. L. Boni, F. Wu, R. Fennimore, and M. M. Batenjany. Preparation of large liposomes by infusion into an aqueous polymer solution. WO0243699 (2002).

5. D. D. Lasic. Recent developments in medical applications of liposomes: Sterically stabilized liposomes in cancer therapy and gene delivery in vivo. J. Controlled Release. 48(2-3):203-222 (1997).

6. R. C. C. New. Preparation of liposomes. In R. C. C. New (ed.), Liposomes: A practical approach, Oxford University Press, New York, 1990, p. 33-104.

7. J. Lasch, V. Weissig, and M. Brandl. Preparation of liposomes. In V. Torchilin, and V. Weissig (eds.), Liposomes: A practical approach, Oxford University Press, New York, 2003, p. 3-29.

8. M. R. Mozafari. Liposomes: An overview of manufacturing techniques. Cell. Mol. Biol. Lett. 10(4):711-719 (2005).

9. A. D. Bangham, M. M. Standish, and J. C. Watkins. Diffusion of univalent ions across the lamellae of swollen phospholipids. $J$. Mol. Biol. 13(1):238-252 (1965).

10. A. D. Bangham, M. M. Standish, and G. Weissmann. The action of steroids and streptolysin S on the permeability of phospholipid structures to cations. J. Mol. Biol. 13(1):253-259 (1965).

11. Y. Ran, and S.H. Yalkowsky. Halothane-a novel solvent toward liposomal formulations for an anticancer agent (AMPB). AAPS Annual Meeting and Exposition, Toronto, Canada, 2002.

12. J. Brunner, P. Skrabal, and H. Hauser. Single bilayer vesicles prepared without sonication. Physico-chemical properties. Biochim. Biophys. Acta. 455(2):322-331 (1976).

13. S. Batzri, and E. D. Korn. Single bilayer liposomes prepared without sonication. Biochim. Biophys. Acta. 298(4):1015-1019 (1973).

14. D. Deamer, and A. D. Bangham. Large volume liposomes by an ether vaporization method. Biochim. Biophys. Acta. 443(3):629634 (1976).

15. D. W. Deamer. Preparation and properties of ether-injection liposomes. Ann. N.Y. Acad. Sci. 308:250-258 (1978).

16. P. Stano, S. Bufali, C. Pisano, F. Bucci, M. Barbarino, M. Santaniello, P. Carminati, and P. L. Luisi. Novel camptothecin analogue (gimatecan)-containing liposomes prepared by the ethanol injection method. J. Liposome Res. 14(1-2):87-109 (2004).

17. S. Hauschild, U. Lipprandt, A. Rumplecker, U. Borchert, A. Rank, R. Schubert, and S. Forster. Direct preparation and loading of lipid and polymer vesicles using inkjets. Small. 1 (12):1177-1180 (2005).

18. C. Li, and Y. Deng. A novel method for the preparation of liposomes: Freeze drying of monophase solutions. J. Pharm. Sci. 93(6):1403-1414 (2004).

19. F. Szoka Jr., and D. Papahadjopoulos. Procedure for preparation of liposomes with large internal aqueous space and high capture by reverse-phase evaporation. P. Natl. Acad. Sci. U.S.A. 75 (9):4194-4198 (1978)

20. S. Frokjaer. Double emulsion vesicles. In R. C. C. New (ed.), Liposomes: A practical approach, Oxford University Press, New York, 1990, p. 68-70.
21. S. Kim, and G. M. Martin. Preparation of cell-size unilamellar liposomes with high captured volume and defined size distribution. Biochim. Biophys. Acta. 646(1):1-9 (1981).

22. M. Takahashi, K.-I. Inafuku, T. Miyagi, H. Oku, K. Wada, T. Imura, and D. Kitamoto. Efficient preparation of liposomes encapsulating food materials using lecithins by a mechanochemical method. J. Oleo Sci. 56(1):35-42 (2007).

23. T. D. Madden, P. R. Harrigan, L. C. L. Tai, M. B. Bally, L. D. Mayer, T. E. Redelmeier, H. C. Loughrey, C. P. S. Tilcock, L. W. Reinish, and P. R. Cullis. The accumulation of drugs within large unilamellar vesicles exhibiting a proton gradient: A survey. Chem. Phys. Lipids. 53(1):37-46 (1990).

24. E. M. Bolotin, R. Cohen, L. K. Bar, N. Emanuel, S. Ninio, D. D. Lasic, and Y. Barenholz. Ammonium sulfate gradients for efficient and stable remote loading of amphipathic weak bases into liposomes and ligandoliposomes. J. Liposome Res. 4(1):455-479 (1994).

25. M. Fan, S. Xu, S. Xia, and X. Zhang. Effect of different preparation methods on physicochemical properties of salidroside liposomes. J. Agric. Food Chem. 55(8):3089-3095 (2007).

26. A. Jahn, W. N. Vreeland, D. L. DeVoe, L. E. Locascio, and M. Gaitan. Microfluidic directed formation of liposomes of controlled size. Langmuir. 23(11):6289-6293 (2007).

27. C. Charcosset, A. El-Harati, and H. Fessi. Preparation of solid lipid nanoparticles using a membrane contactor. J. Controlled Release. 108(1):112-120 (2005).

28. K. Anton, P. Van Hoogevest, and L. Frederiksen. Preparation of a liposome dispersion containing an active agent by compressiondecompression. EP616801 (1994).

29. K. Otake, T. Imura, H. Sakai, and M. Abe. Development of a new preparation method of liposomes using supercritical carbon dioxide. Langmuir. 17(13):3898-3901 (2001).

30. T. P. Castor, and L. Chu. Methods and apparatus for making liposomes containing hydrophobic drugs. US5776486 (1998).

31. R. T. Bustami, H.-K. Chan, F. Dehghani, and N. R. Foster. Recent applications of supercritical fluid technology to pharmaceutical powder systems. Kona. 19:57-70 (2001).

32. T. P. Castor. Methods and apparatus for liposomes preparation. WO9427581 (1994).

33. T. P. Castor, and L. Chu. Methods and apparatus for making liposomes containing hydrophobic drugs. WO9615774 (1996).

34. T. P. Castor. Phospholipid nanosomes. Current Drug Delivery. 2 (4):329-340 (2005).

35. L. Frederiksen, K. Anton, P. van Hoogevest, H. R. Keller, and $\mathrm{H}$. Leuenberger. Preparation of liposomes encapsulating watersoluble compounds using supercritical carbon dioxide. J. Pharm. Sci. 86(8):921-928 (1997).

36. L. Frederiksen, K. Anton, B.J. Barratt, P. Van Hoogevest, and H. Leuenberger. Use of supercritical carbon dioxide for preparation of pharmaceutical formulations. 3rd International Symposium on Supercritical Fluids, vol. 3, Tome, Strasbourg, France, 1994, pp. $235-240$

37. R.H. Bridson, R.C.D. Santos, B. Al-Duri, S.M. McAllister, J. Robertson and H.O. Alpar. The preparation of liposomes using compressed carbon dioxide: Strategies, important considerations and comparison with conventional techniques. J. Pharm. Pharmacol. 58(6):775-785 (2006)

38. T. Imura, K. Otake, S. Hashimoto, T. Gotoh, M. Yuasa, S. Yokoyama, H. Sakai, J. F. Rathman, and M. Abe. Preparation and physicochemical properties of various soybean lecithin liposomes using supercritical reverse phase evaporation method. Colloid Surface B. 27(2-3):133-140 (2002).

39. T. Imura, T. Gotoh, K. Otake, S. Yoda, Y. Takebayashi, S. Yokoyama, H. Takebayashi, H. Sakai, M. Yuasa, and M. Abe. Control of physicochemical properties of liposomes using a supercritical reverse phase evaporation method. Langmuir. 19 (6):2021-2025 (2003).

40. K. Otake, T. Imura, S. Yoda, Y. Takebayashi, T. Sugeta, N. Nakazawa, H. Sakai, and M. Abe. Formation and physicochemical properties of liposomes using a supercritical reverse phase evaporation method. 6th International Symposium on Supercritical Fluids, Versailles, France, 2003.

41. K. Otake, T. Shimomura, T. Goto, T. Imura, T. Furuya, S. Yoda, Y. Takebayashi, H. Sakai, and M. Abe. Preparation of liposomes using an improved supercritical reverse phase evaporation method. Langmuir. 22(6):2543-2550 (2006). 
42. K. Otake, T. Shimomura, T. Goto, T. Imura, T. Furuya, S. Yoda, Y. Takebayashi, H. Sakai, and M. Abe. One-step preparation of chitosan-coated cationic liposomes by an improved supercritical reverse-phase evaporation method. Langmuir. 22(9):4054-4059 (2006).

43. C. Magnan, E. Badens, N. Commenges, and G. Charbit. Soy lecithin micronization by precipitation with a compressed fluid antisolvent- influence of process parameters. J. Supercrit. Fluids. 19(1):69-77 (2000).

44. E. Badens, C. Magnan, and G. Charbit. Microparticles of soy lecithin formed by supercritical processes. Biotechnol. Bioeng. 72 (2):194-204 (2001).

45. S. Kunastitchai, L. Pichert, N. Sarisuta, and B. W. Muller. Application of aerosol solvent extraction system (ASES) process for preparation of liposomes in a dry and reconstitutable form. Int. J. Pharm. 316(1-2):93-101 (2006).

46. S. Kunastitchai, N. Sarisuta, B. Panyarachun, and B. W. Muller. Physical and chemical stability of miconazole liposomes prepared by supercritical aerosol solvent extraction system (ASES) process. Pharm. Dev. Technol. 12(4):361-370 (2007).

47. A. Nakajima, C. Nagaike, and E. Ueda. Manufacture of liposome lipids by using supercritical or subcritical carbon dioxide. JP2005162678 (2005).

48. C. Nagaike, and Y. Motokui. Method of producing liposomecontaining preparation. WO2006016468 (2006).

49. Y. Aoki, and E. Ueda. Liposome compositions for cancer therapy, and manufacture thereof. JP2006063009 (2006).

50. E. Ueda. Ultrasound imaging agent containing liposomes, and production thereof. JP2006063052 (2006).
51. E. Ueda, A. Nakajima, K. Motokui, and C. Nagaike. Manufacture of liposomes for target delivery. JP2006069930 (2006)

52. Y. Motokui, and T. Wada. Method for production of liposome compositions by using supercritical fluid carbon dioxide. JP2006298842 (2006)

53. T. Wada, and Y. Motokui. Liposome compositions containing X-ray contrast agents, and production thereof. JP2006298845 (2006).

54. B. Yan, X.-Q. An, J. Bai, and Y.-H. Zhang. Preparation of cefazolin sodium liposomes by supercritical carbon dioxide method. Wuli Huaxue Xuebao. 22(2):226-229 (2006).

55. L. A. Meure. The development of a novel process for the formation of liposomes: Depressurisation of an expanded solution into aqueous media (DESAM), PhD Thesis, University of New South Wales, Sydney, Australia, 2004.

56. A. Wagner, M. Platzgummer, G. Kreismayr, H. Quendler, G. Stiegler, B. Ferko, G. Vecera, K. Vorauer-Uhl, and H. Katinger. Gmp production of liposomes- a new industrial approach. $J$. Liposome Res. 16(3):311-319 (2006).

57. A. K. Dillow, F. Dehghani, J. S. Hrkach, N. R. Foster, and R. Langer. Bacterial inactivation by using near-and supercritical carbon dioxide. Proc. Natl. Acad. Sci. U. S. A. 96(18):1034410348 (1999).

58. J. Li, G.L. Thompson, M.A. Matthews, and T.A. Davis. Dense carbon dioxide for cleaning biomaterials: removing $S$. aureus and bacterial endotoxins. AIChE Annual Meeting, San Francisco, USA, 2003.

59. J. Zhang, T.R. Thomas, T.A. Davis, and M.A. Matthews, Sterilization of bacterial spores with high-density carbon dioxide. AIChE Annual Meeting, San Francisco, USA, 2003. 